\title{
Towards a Tilt-to-Length Coupling Calibration on the GRACE Follow-On Laser Ranging Instrument
}

\author{
Henry Wegener (AEI Hannover, Germany) \\ on behalf of the LRI team
}

October 11, 2018 


\section{Overview}

1. Introduction to the GRACE Follow-On (GFO) LRI

2. LRI measurement principle

3. Laser link acquisition

4. Tilt-to-Length (TTL) coupling

5. GFO attitude control \& attitude sensing 


\section{Introduction}

- GRACE (2002 - 2017)

- very successful LEO satellite-pair measuring Earth's gravity field

- main observable: biased range from K-Band Ranging (KBR)

- GRACE Follow-On (GFO; launch: 22 May 2018)

- almost identical copy of GRACE

- main observable: KBR range (same as on GRACE)

- Laser Ranging Instrument (LRI)

- technology demonstrator

- first laser interferometer measuring inter-satellite range ever operated in space

- initial turn-on: 13 June 2018 


\section{LRI measurement principle}

The LRI is a heterodyne optical interferometer

- operated with two $1064 \mathrm{~nm}$ NPRO lasers

- arranged in a race-track configuration

- with an offset phase-locked transponder scheme

- designed for phase-tracking of beatnote frequencies from 4 to $16 \mathrm{MHz}$

- measuring the biased range between the GFO satellites over a distance of $220 \mathrm{~km}$ 


\section{LRI measurement principle}

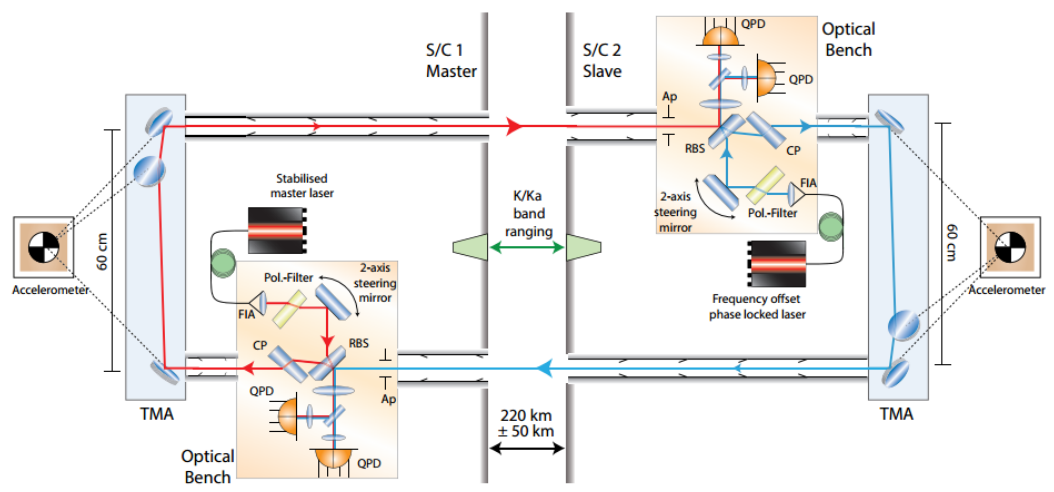

Figure: Sketch of the LRI measurement principle. Image credit: Vitali Müller / AEI Hannover 


\section{Initial Acquisition}

- Due to initial uncertainties (e.g. alignment tolerances), the LRI had to perform an initial acquisition $\operatorname{scan}^{1}$ with

- 9 hour duration

- 5 degrees of freedom: pitch and yaw angles (both S/C), slave laser frequency

- When all 5 parameters are very close to optimal, a flash is seen on the master photo diode

- The recorded data is sent to ground and analyzed

- Parameter files are uploaded to the S/C

- LRI goes into re-acquisition mode and then science mode

1. Koch et al (AEI Hannover) - Line of sight calibration for the laser ranging interferometer on-board the GRACE Follow-On mission: on-ground experimental validation 


\section{Initial Acquisition}

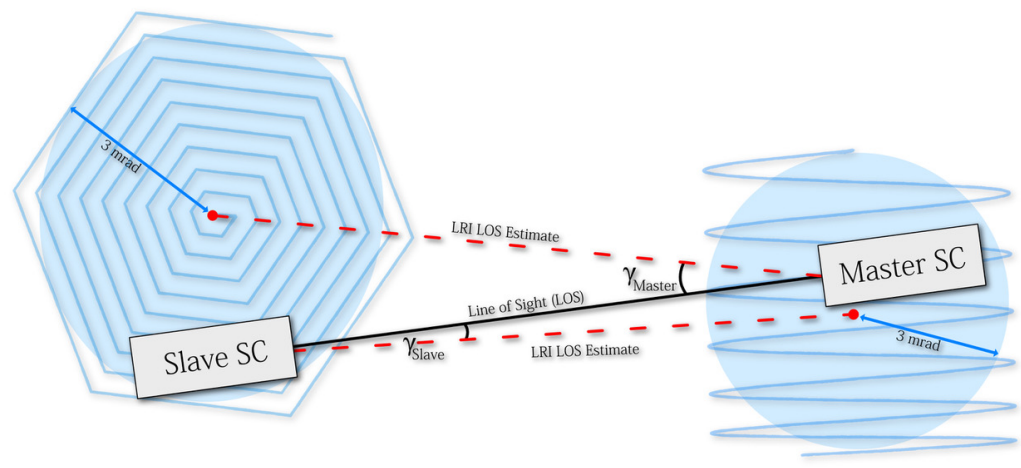

Figure: Schematic of initial acquisition scan. Image credit: Alexander Koch / AEI Hannover 


\section{GRACE Follow-On LRI: first light !}

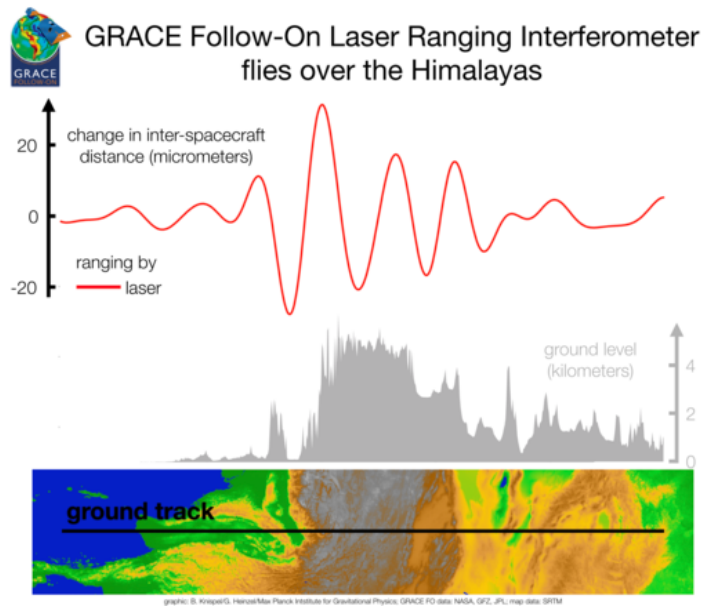

Figure: LRI - Himalaya plot. Image credit: Gerhard Heinzel / AEI Hannover 


\section{GRACE Follow-On LRI: first light !}

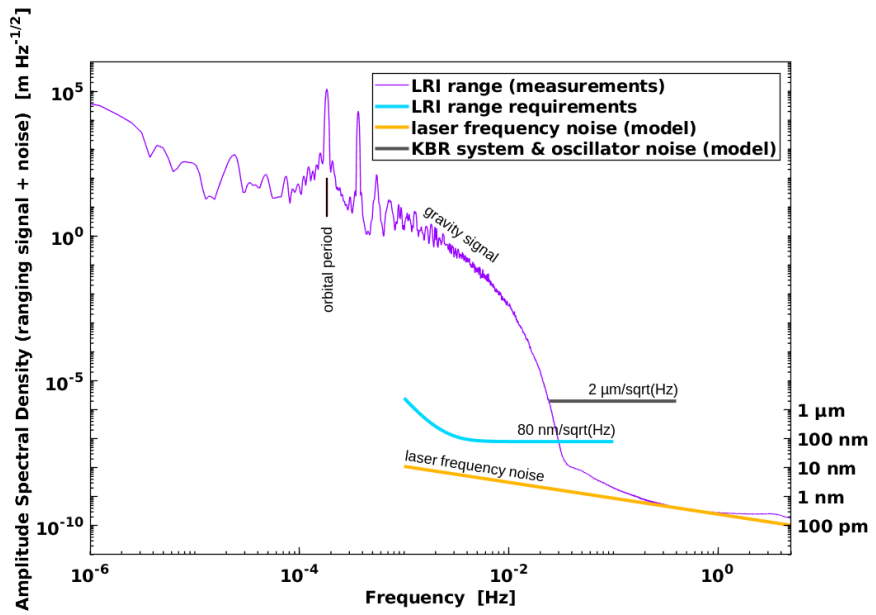

Figure: LRI - ranging spectrum (10 day segment, including all signal and noise) 


\section{LRI Tilt-to-Length Coupling}

- Tilt-to-Length (TTL) coupling is the undesired propagation of satellite pointing jitter into the ranging measurement

- can be assumed linear in the pointing angles (roll, pitch, yaw)

- coupling factors are the scales of the ranging error caused by each pointing angle

- TTL calibration means to determine these 6 coupling factors, in order to subtract the error from the measurement

- Among several other effects, the main reason for TTL coupling is an unknown offset between the vertex point (VP) of the TMA and the satellite center-of-mass (CoM) 


\section{LRI Tilt-to-Length Coupling}

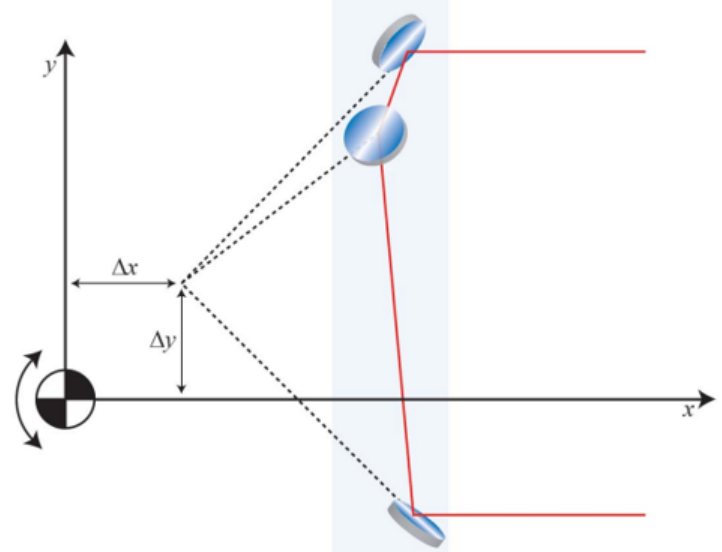

Figure: Offset between TMA vertex point and satellite CoM 


\section{TTL calibration}

TTL coupling can be calibrated

- using center-of-mass calibration (CMC) maneuvers

- satellite rotation maneuver

- using magnetic torque rods

- performed on GRACE and GFO to calibrate the accelerometer

- using dedicated calibration maneuver

- similar to the CMC

- with adjusted maneuver parameters

- using regular data stream

- (+) long time series available

- (-) complicated data analysis 


\section{GRACE Follow-On: Attitude Control}

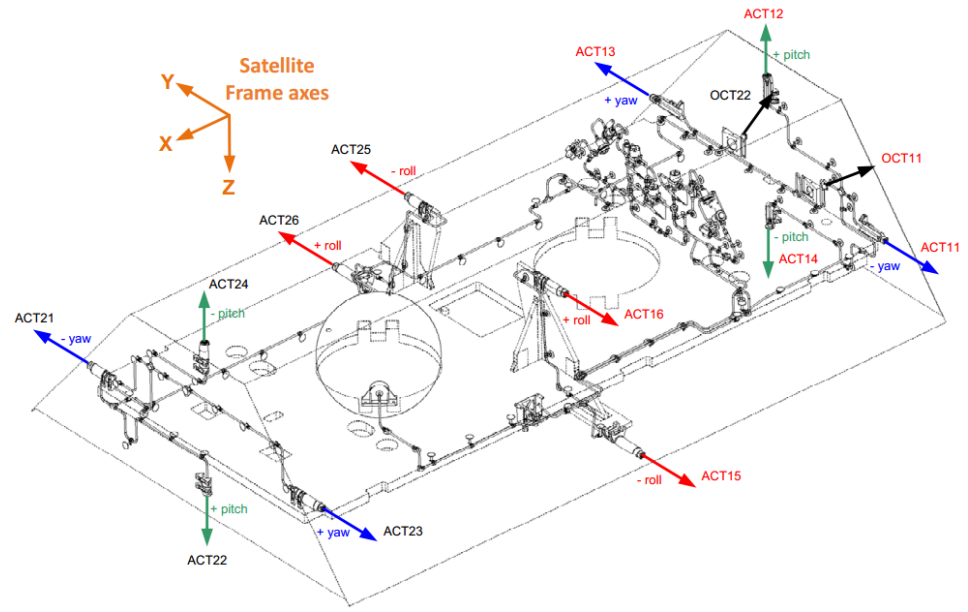

Figure: GRACE Follow-On attitude control thruster accomodation. Image credit: NASA JPL 


\section{GRACE Follow-On: Attitude Control}

- same principle as on GRACE

- six magnetic torque rods (max. $110 \mathrm{~mA}$ or $27.5 \mathrm{Am}^{2}$ )

- twelve attitude control thrusters $\left(N_{2}, 10 \mathrm{mN}\right)$

- smaller deadbands for thruster control:

- 2500 / 250 / $250 \mu \mathrm{rad}$ (roll / pitch / yaw)

- total mass increased from $475 \mathrm{~kg}$ to $655 \mathrm{~kg}$

$$
J_{\text {GRACE }}=\left(\begin{array}{ccc}
70.23 & -3.0 & -2.883 \\
-3.0 & 345.14 & -0.348 \\
-2.883 & -0.348 & 388.84
\end{array}\right) \mathrm{kg} \mathrm{m}^{2} \quad J_{\text {GRACE-FO C }}=\left(\begin{array}{ccc}
110.4 & 0.9 & 0.3 \\
0.9 & 580.5 & 0.03 \\
0.3 & 0.03 & 649.5
\end{array}\right) \mathrm{kg} \mathrm{m}^{2}
$$




\section{GRACE Follow-On: Attitude Determination}

- IMU, SCA, DWS / FSM, ACC(, CESS)

- 4(5) sensors measuring different quantities

- SCA Level-1B data planned to contain quaternions from Kalman filter solution (according to Level1DataProductUserHandbook)

- improvement: much more sensors than on GRACE

- expected lower noise attitude solutions 


\section{GRACE Follow-On: Attitude Determination}
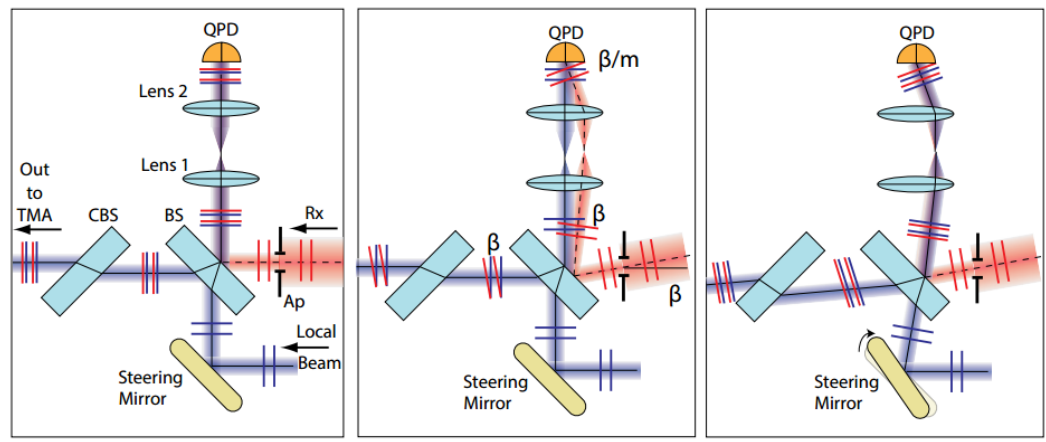

Figure: Differential Wavefront Sensing (DWS) principle 


\section{Acknowledgements}

\section{Thank you for your attention !}

\section{Acknowledgements}

- The LRI is jointly managed by NASA JPL, and the AEI Hannover

- This work is presented on behalf of the LRI team

- Thanks to Bundesministerium für Bildung und Forschung (BMBF) for financial support (FKZ 03F0654B)

- Thanks to DFG Sonderforschungsbereich (SFB) 1128 Relativistic Geodesy and Gravimetry with Quantum Sensors (geo-Q) for financial support 